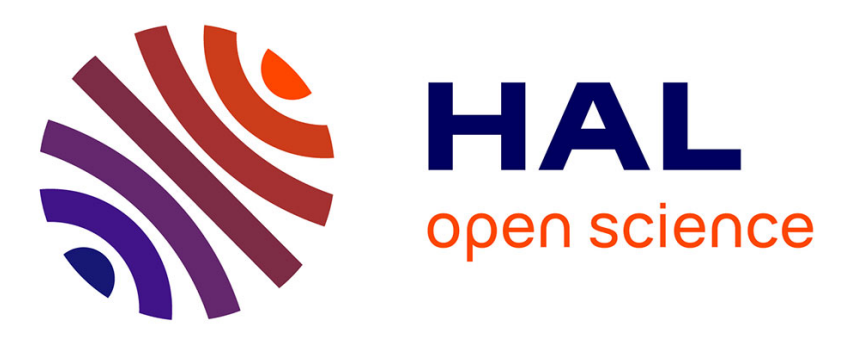

\title{
Position-based modeling of lesion displacement in Ultrasound-guided breast biopsy
}

Eleonora Tagliabue, Diego Dall'alba, Enrico Magnabosco, Chiara Tenga, Igor Peterlík, Paolo Fiorini

\section{- To cite this version:}

Eleonora Tagliabue, Diego Dall'alba, Enrico Magnabosco, Chiara Tenga, Igor Peterlík, et al.. Positionbased modeling of lesion displacement in Ultrasound-guided breast biopsy. International Journal of Computer Assisted Radiology and Surgery, 2019, 14 (8), pp.1329-1339. 10.1007/s11548-019-01997-z . hal-02276090

\section{HAL Id: hal-02276090 https://hal.science/hal-02276090}

Submitted on 2 Sep 2019

HAL is a multi-disciplinary open access archive for the deposit and dissemination of scientific research documents, whether they are published or not. The documents may come from teaching and research institutions in France or abroad, or from public or private research centers.
L'archive ouverte pluridisciplinaire HAL, est destinée au dépôt et à la diffusion de documents scientifiques de niveau recherche, publiés ou non, émanant des établissements d'enseignement et de recherche français ou étrangers, des laboratoires publics ou privés. 


\title{
Position-based modeling of lesion displacement in Ultrasound-guided breast biopsy
}

\author{
Eleonora Tagliabue · Diego Dall'Alba · Enrico \\ Magnabosco - Chiara Tenga · Igor Peterlik . \\ Paolo Fiorini
}

Received: 10 January 2019 / Accepted: 13 May 2019

\begin{abstract}
Purpose Although ultrasound (US) images represent the most popular modality for guiding breast biopsy, malignant regions are often missed by sonography, thus preventing accurate lesion localization which is essential for a successful procedure. Biomechanical models can support the localization of suspicious areas identified on a pre-operative image during US scanning since they are able to account for anatomical deformations resulting from US probe pressure. We propose a deformation model which relies on position-based dynamics (PBD) approach to predict the displacement of internal targets induced by probe interaction during US acquisition.
\end{abstract}

Methods The PBD implementation available in NVIDIA FleX is exploited to create an anatomical model capable of deforming online. Simulation parameters are initialized on a calibration phantom under different levels of probe-induced deformations, then they are fine-tuned by minimizing the localization error of a US-visible landmark of a realistic breast phantom. The updated model is used to estimate the displacement of other internal lesions due to probe-tissue interaction.

Results The localization error obtained when applying the PBD model remains below 11 $\mathrm{mm}$ for all the tumors even for input displacements in the order of $30 \mathrm{~mm}$. This proposed method obtains results aligned with FE models with faster computational performance, suitable for real-time applications. In addition, it outperforms rigid model used to track lesion position in US-guided breast biopsies, at least halving the localization error for all the displacement ranges considered.

E. Tagliabue $\cdot$ D. Dall'Alba $\cdot$ E. Magnabosco $\cdot$ C. Tenga $\cdot$ P. Fiorini

Dept. of Computer Science, University of Verona, Str. le Grazie 15, Verona (Italy)

E-mail: eleonora.tagliabue@univr.it

I. Peterlik

INRIA, Strasbourg (France) 
Conclusions Position-based dynamics approach has proved to be successful in modeling breast tissue deformations during US acquisition. Its stability, accuracy and real-time performance make such model suitable for tracking lesions displacement during US-guided breast biopsy.

Keywords Biomechanical model · Position-based dynamics · Ultrasound-guided breast biopsy · Ultrasound tracking

\section{Introduction}

Image-guided breast biopsy is the standard procedure to evaluate symptomatic and screeningdetected suspicious lesions [1]. Among all types of image-guided breast biopsies that can be performed, ultrasound (US) guidance is widely preferred since it allows to reach difficult areas while optimizing procedure time, without exposing the patient to any harmful radiation, thus overcoming the limitations of biopsies performed under Magnetic Resonance Imaging (MRI) and X-rays guidance. However, besides the fact that procedure outcome strongly depends on clinicians' expertise and available equipment, certain malignant lesions are often challenging to be distinguished in US [2]. Recently, image fusion techniques have been investigated to create navigable anatomy reconstructions that enable the visualization of MRI-detected lesions on real-time US images [3]. Both commercial and research platforms that implement this strategy align the two images by computing either rigid or affine transformation which minimizes the matching error between sets of corresponding landmarks $[4,5]$. The main limitation of this approach is that it does not account for the highly deformable nature of the breast. Typically, MRI acquisition is performed with the patient in prone position, whereas the subject lies semi-supine during US scanning. During this repositioning, the breast undergoes large deformations. In addition, in order to obtain acceptable image quality, the physician applies compression forces on the breast with the US probe to guarantee a proper coupling. Ideally, MRI-US fusion should be able to account for these deformations in order to accurately track the motion of internal targets.

Due to the need of modelling these gross deformations, the development of models able to realistically describe breast behavior in clinical settings remains an active research field. Many of these methods focus on the definition of an a-priori deformation model of the structure of interest. The 3D geometry of the anatomy is extracted from the MRI and initialized with known elastic properties and/or parameters, and it is later used to predict structures displacements and deformations, given certain inputs. These models have been employed for co-registration between MRI and other modalities but never with 2D US [6]. This approach has the potential to accurately predict the displacement of MRI-detected lesions even in cases where target areas cannot be identified on US.

The most popular numerical procedure capable of achieving mechanically realistic simulations of the breast relies on the finite element method (FEM) [6]. FEM describes soft tissues through a continuum mechanics formulation and determines future positions by solving physical balance laws. However, high accuracy is obtained at the expense of high complexity, which makes the solution method computationally expensive, thus not generally compatible with real-time. It can happen that the simplifications introduced in the solving process are so strict that the results obtained are less accurate than expected and may even lose their physical meaning [7]. These limitations of FEM can be overcome by geometrybased approaches, like the position-based dynamics (PBD). Instead of predicting volume deformations based on time integration of Newton's second law, as in FEM-based simulations, the PBD approach models objects as an ensemble of particles whose positions are 
directly updated, as a solution of a quasi-static problem subject to geometrical constraints [8]. This makes it possible both to achieve real-time performances and to avoid 3D meshing process, since particles are placed in space to fill a surface-delimited volume. Stability, robustness and simplicity are among the main reasons for the increasing popularity of the PBD method [8]. Even though the time evolution of the system does not depend on the integration of physical laws based on real mechanical parameters, some works showed that different types of properties and behaviours of elastic materials can be simulated with PBD by appropriately manipulating modelling parameters and constraint functions of the system $[9,10]$.

Although the PBD approach has been mainly applied in computer graphic fields, the enhanced speed, controllability and unconditional stability of this method are its most appealing features for its application to medical simulation as well, where interactions between multiple organs and tools have to be modelled and solved in real-time. The most popular exploitation of the PBD concept in the medical field has been in the development of training simulator for surgical procedures involving dissection, since PBD methods are able to handle topological changes involved in such tasks maintaining real-time interactive performance $[11,12]$. Some other works have focused on the achievement of particular types of deformations by coupling the PBD formulation with mass-spring models [13, 14] or for the simulation of knot tying procedure [15]. Camara et al. employed the PBD scheme to create a patient-specific biomechanical model of the kidney for the real-time simulation of intra-operative US images [16]. Differently from the works mentioned above, here the authors tuned the most relevant PBD parameters to obtain an accurate simulation. They started from the results obtained in their previous work, where PBD parameters were calibrated by minimizing the distance between real and simulated fiducial positions over a sequence of 3 deformations induced by a US probe pressing on a porcine kidney [17]; then, they refined such parameters to account for the different material properties of the kidney phantom used.

We propose a method that relies on the position-based dynamics (PBD) concept to approximate the motion of internal structures during US scanning. To the best of our knowledge, this is the first work where probe-induced deformations are taken into account in real time. Starting from the work in [17], we initialize PBD parameters with those estimated on a deformable calibration phantom resembling the scenario of interest, subject to several different probe-induced deformations. As a following step, we fine-tune such properties on the anatomy of interest by tracking the displacement of one US-visible landmark. The fine-tuning procedure allows to customize model parameters and obtain a patient-specific deformation model that can predict in real-time the displacement of other internal areas, even if they are visible only on the initial MRI, thus improving lesion tracking and targeting during biopsy procedures. The proposed PBD model makes it possible to achieve an accurate and stable simulation of large deformations without even requiring a complex 3D mesh generation procedure (which is needed for FEM), which makes the model easy to be generalized and applied directly to the clinical scenario.

The rest of this paper is structured as follows. In Section 2 we introduce the main principles of the position-based dynamics formulation and we present the setup used for the experiments. Section 3 reports results relative to the calibration and validation phases, which are discussed in Section 4. Finally, Section 5 presents our conclusions. 


\section{Methods}

\subsection{Position-based dynamics approach}

Position-based dynamics is a simulation approach that computes the time evolution of a dynamic system by directly updating positions, as first described by Müller et al. in [18]. Simulated objects are discretized as sets of particles, described by their positions $\mathbf{p}_{i}$ and velocities $\mathbf{v}_{i}$, subject to a set of positional constraints $C_{j}\left(\mathbf{p}_{1}, \ldots, \mathbf{p}_{n}\right) \succ 0$ (symbol $\succ$ denotes either $=$ or $\geq$ ). In the PBD approach, deformation calculation becomes a constraint-function optimization problem. The simulation workflow starts with a prediction step in which simplectic Euler integration is performed to guess new particle positions and velocities. Then, non-linear Gauss-Seidel solver is used to find the correction $\Delta \mathbf{p}$ to apply to the estimated positions in an iterative fashion, so that each constraint equation (after linearization) is individually satisfied:

$$
C(\mathbf{p}+\Delta \mathbf{p}) \approx C(\mathbf{p})+\nabla C(\mathbf{p}) \Delta \mathbf{p} \succ 0
$$

Since the resulting system is under-determined, the position update $\Delta \mathbf{p}$ is constrained to ensure the preservation of linear and angular momenta, which corresponds to forcing $\Delta \mathbf{p}$ to lie in the direction of the constraint gradient $\nabla C$. The position update is further weighted by the inverse of the mass matrix $\mathbf{M}$ and multiplied by a parameter $k \in[0,1]$ which represents the stiffness of the constraint:

$$
\Delta \mathbf{p}=k \lambda \mathbf{M}^{-1} \nabla C(\mathbf{p})^{T}
$$

The Lagrange multiplier $\lambda$ which solves Equation 1 is thus unique and given by:

$$
\lambda=\frac{C(\mathbf{p})}{\Delta C(\mathbf{p}) \mathbf{M}^{-1} \Delta C(\mathbf{p})^{T}}
$$

Finally, computed $\Delta \mathbf{p}$ are used to correct both the positions and the velocities.

From this implementation, it follows that simulation behavior and performance are not only influenced by the relative position, dimension and number of particles in space, but also by the constraints acting among particles. For example, large deformations of soft bodies are usually achieved by defining positional constraints among rigid clusters of adjacent particles. This kind of constraint is called region-based shape matching. For all the particles which lie within a cluster, goal positions $\mathbf{g}_{i}$ are determined after estimating the optimal transformation $\mathbf{T}$ that matches initial and deformed positions (denoted by $\mathbf{p}_{i}^{0}$ and $\mathbf{p}_{i}$, respectively) in a leastsquare fashion:

$$
\mathbf{g}_{i}=\mathbf{T}\left(\begin{array}{c}
\mathbf{p}_{i}^{0} \\
1
\end{array}\right)
$$

Since clusters can overlap (i.e., particles may belong to multiple clusters) the final goal position for a particle is obtained by averaging goal positions of the corresponding regions. Position corrections are then computed as:

$$
\Delta \mathbf{p}_{i}=\alpha\left(\mathbf{g}_{i}-\mathbf{p}_{i}\right)
$$

where $\alpha \in[0,1]$ is the stiffness used to enforce the constraint. As a consequence, realistic elastic behavior is obtained by appropriately selecting cluster parameters. For example, the higher the number of clusters, the more degrees of freedom the body will have.

Efficient implementations of the PBD approach together with the region-based shape matching constraint are currently available in several software libraries, such as the NVIDIA FleX [19]. 


\subsection{Experimental Setup}

The experimental data are acquired from a Freehand Ultrasound System (FUS) based on a Telemed MicrUs US device (Telemed, Vilnius, Lithuania) equipped with a linear probe (model L12-5N40) and an optical tracking system MicronTracker Hx40 (ClaronNav, Toronto, Canada) (Figure 1). We performed all experiments with an acquisition frequency of $5 \mathrm{MHz}$ and a depth setting of $50 \mathrm{~mm}$, while all the other parameters are kept to default values provided by manufacturer. The spatial and temporal calibration methods used in the study are based on the PLUS toolkit, a software and hardware framework for building research FUS [20]. The overall probe spatial calibration error is below $1 \mathrm{~mm}( \pm 0.7147)$, and below $0.5 \mathrm{~mm}$ $( \pm 0.334)$ for the pointer used for fiducial points localization required for the rigid registration. Thanks to the FUS, we can know in real-time the position and orientation of the US image plane and therefore extract three-dimensional position of any pixel belonging to the image.

Data visualization and analysis are performed in 3D Slicer, e.g. landmarks-based rigid registration between CT-extracted 3D models and FUS reference system or landmark localization and tracking in US images [21]. The simulation environment was developed in Unity 2018.3 using NVIDIA FleX on a workstation equipped with an AMD Ryzen 71600 processor, 16GB RAM and a Titan Xp GPU donated by NVIDIA Corporation. The communication between FUS (i.e. US images and tracked objects positions and orientations) and other software modules is based on OpenIGTLink protocol [22].

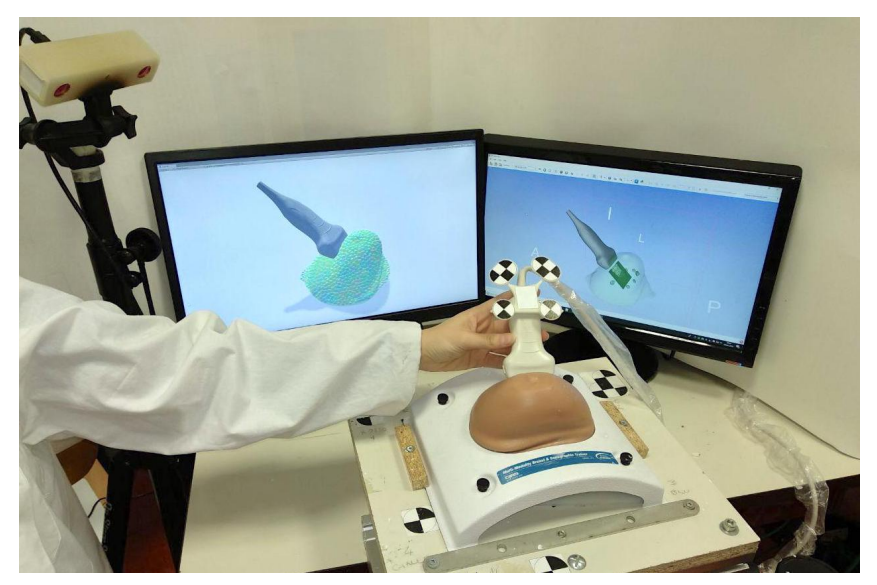

Fig. 1 The FUS system allows to map the real positions of the CIRS breast phantom and the US probe to the 3D Slicer scene (right monitor). Information about probe spatial transformation is communicated to the simulated environment in Unity (left monitor)

\subsubsection{Calibration phantom}

Simulation parameters of the developed deformation model are initialized on a handcrafted box-shaped calibration phantom $(155 \times 100 \times 70 \mathrm{~mm})$, made of ballistic gel as described in [23]. In addition to correctly approximating the consistency of the clinical scenario of interest, ballistic gel also has realistic echogenic properties. The realism of the setup is further 
enhanced with the inclusion of three stiffer beads with a diameter of $18 \mathrm{~mm}$ within the phantom, placed at three different heights $(47 \mathrm{~mm}, 53 \mathrm{~mm}, 63 \mathrm{~mm})$. The main advantage of performing an initial calibration on a minimalistic phantom is that it provides a reasonable starting guess of deformation parameters, common for all the patients. We expect that such parameters will not be able to model each anatomy, and a subject-specific tuning of their values will be necessary to account for inter-patient variability. However, having an initial acceptable guess of the parameters will allow to start the refinement process from a point closer to the optimum and also to restrict the search space, making the pre-operative optimization more efficient. Figure 2 shows the corresponding simulation environment, where the virtual US probe is modelled as a rigid body which follows in real-time its physical tracked counterpart. Probe-tissue interaction is modelled as a contact problem, handled by the default collision detection and response implementation provided by the Unity engine. As boundary condition for the simulation, we fixed all the points which belong to the lowest phantom surface. Despite the presence of stiffer internal parts, the proposed model treats

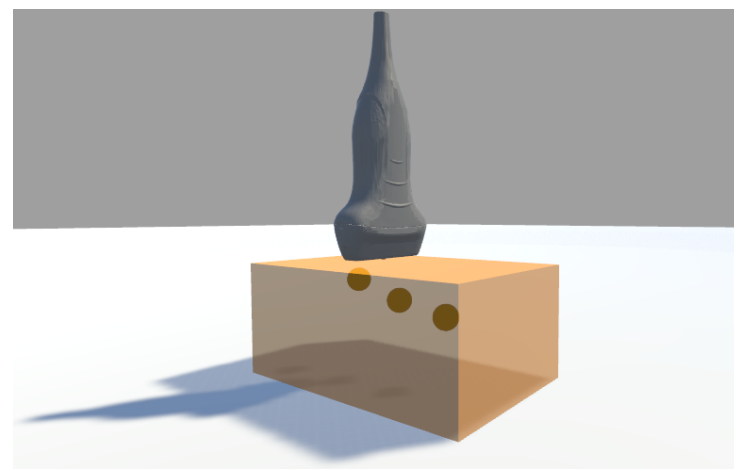

Fig. 2 Calibration phantom in the simulated scene in Unity

the deformable object as homogeneous and, as a consequence, identifies global parameters. The reason for this choice resides in the fact that, since PBD simulation parameters do not have a direct physical meaning, identification of those that describe heterogeneous material properties would not have been straightforward and would require a thorough study.

In general, PBD simulations are controlled by a high number of parameters, but tuning all of them is out of the scope of this research. In the calibration procedure, we focus on the optimization of the parameters defining the clusters of region-based shape matching constraint present in Nvidia FleX implementation, which control objects' deformable behavior: cluster spacing (i.e., the distance between adjacent clusters), cluster radius (i.e., the radius of each cluster region) and cluster stiffness (i.e., the extent to which adjacent cluster are constrained to each other). Although it is well known that other PBD parameters can have an impact on soft body behavior, we decide to keep their values fixed for all the simulations and to set them in accordance with previous works (Table 1) [17]. The experimental protocol followed for parameters optimization consists of five acquisitions for each phantom inclusion. The initial rest condition is obtained by only slightly touching the phantom with the US probe, without inducing any deformation. Afterwards, four US images are acquired in correspondence of the center of each bead, by applying downward probe displacements of $5 \mathrm{~mm}, 10 \mathrm{~mm}, 15 \mathrm{~mm}$ and $20 \mathrm{~mm}$. Estimation of optimal model parameters for the calibration phantom is performed with the genetic algorithm scheme. By generating a population of 
Table 1 PBD parameters kept constant for all the simulations

\begin{tabular}{lr}
\hline Parameter & Value \\
\hline Time step & $0.02 \mathrm{~s}$ \\
Simulation substeps & 3 \\
Substep iterations & 9 \\
Relaxation type & Local \\
Gravity & $9.81 \mathrm{~m} / \mathrm{s}^{2}$ \\
Volume sampling & 7 \\
Particle spacing & $5 \mathrm{~mm}$ \\
Shape friction coefficient & 0.35 \\
Particle friction coefficient & 0.25 \\
Damping factor & 12 \\
Collision distance & $3 \mathrm{~mm}$ \\
Self-collision & True \\
\hline
\end{tabular}

possible solutions at each iteration in a stochastic way, this methodology eventually evolves towards an optimal solution. This scheme is known for being able to offer good characteristics of exploration and exploitation of the search space [24]. In this work, we rely on the implementation provided in MATLAB (MATLAB R2018b, Mathworks, Natick, MA, USA). We minimize the prediction error, formulated by the following bound-constrained problem:

$$
\mathbf{p}^{*}{ }_{g e l}=\underset{\mathbf{l b}<\mathbf{p}<\mathbf{u b}}{\arg \min } \sum_{n=1}^{N} \sum_{l=1}^{L}\left\|\mathbf{X}_{\mathbf{P B D}}(\mathbf{p}, l, n)-\mathbf{X}_{\mathbf{U S}}(l, n)\right\|
$$

where $\mathbf{p}$ is the vector of parameters to optimize, $\mathbf{X}_{\mathbf{P B D}}(\mathbf{p}, l, n)$ is the position of the tracked PBD particle, $\mathbf{X}_{\mathbf{U S}}(l, n)$ is the reference bead position, both relative to bead $n$ at deformation level $l$, and the symbol $\|$.$\| represents the Euclidean distance. \mathbf{X}_{\mathbf{U S}}(l, n)$ are selected on US images as points belonging to bead contours which lie closer to the US probe; this choice is motivated by the better visibility of interfaces on US, and allows to avoid additional inaccuracies induced by image segmentation necessary, for example, for centroid computation. The tracked PBD particle (which gives $\mathbf{X}_{\mathbf{P B D}}(\mathbf{p}, l, n)$ ) is chosen as the one with minimum distance from the point on bead contour closest to the US probe, in the rest configuration. In order to describe the entire model as deformable, we constrain all the particles to fall within at least one cluster by imposing cluster radius to be at least half of cluster spacing. Lower and upper bounds (lb, ub) for cluster spacing parameter are set to $[5 ; 35] \mathrm{mm}$, where the minimum value is constrained by particle spacing (Table 1), while the maximum value corresponds to half of the smallest gel dimension. Consequently, cluster radius is restricted to the range $[2.5 ; 15] \mathrm{mm}$. Instead, cluster stiffness is left free to vary within the entire acceptable range $[0 ; 1]$.

\subsubsection{Breast phantom}

The biomechanical model presented aims at predicting the displacement of internal lesions detected pre-operatively that follows from the pressure applied by the US probe. In this work, a realistic multi-modality breast phantom (Model 073; CIRS, Norfolk, VA, USA) is used to evaluate the capability of the PBD model to provide correct estimates of biopsy targets. The 3D model of the CIRS phantom's external surface and 10 inner lesions (diameter of $5-10 \mathrm{~mm}$ ) is obtained by segmenting the corresponding CT image relying on the active contour approach implemented in ITK-SNAP framework [25], and they are later simplified (smoothed and decimated) without affecting the topology, using MeshLab [26] (Figure 3). 


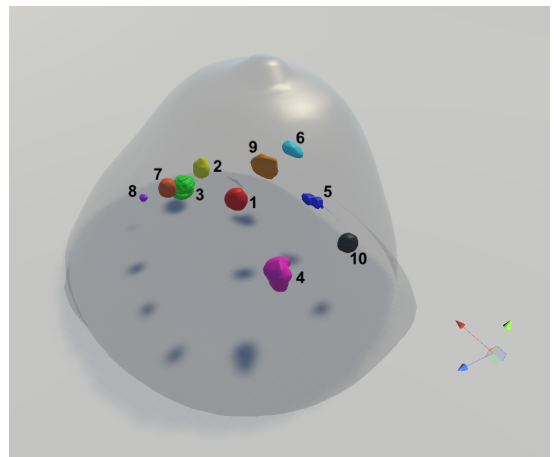

(a)

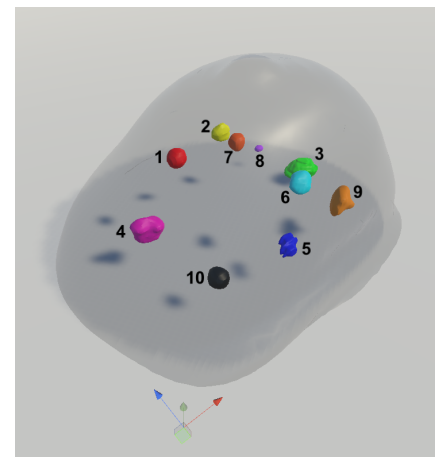

(b)

Fig. 3 External surface and inner lesions of the CIRS breast phantom in two different views

Coherently with the previous setup, we constrain the motion of all the points belonging to the phantom base. Moreover, gravitational force is not applied in this simulation, since the geometry model already represents the phantom in a gravity-loaded configuration. The PBD model of the clinical scenario of interest shares the same fixed parameters of Table 1. A minimalistic scene of the simulated environment in Unity is made available ${ }^{1}$. In order to obtain a patient-specific simulation, some experiments are conducted to refine the values of cluster spacing, radius and stiffness parameters before applying the model to predict lesions displacement due to US probe interaction. This process, which we refer to as fine tuning, consists of tracking the position of a single US-visible landmark subject to four probe-induced deformations $(15,20,25,30 \mathrm{~mm})$ in a similar fashion to what has been done for the calibration phantom (Figure 4). In our case, lesion labelled with number 1 is used as a reference for this procedure. It is worth stressing out that the choice of a lesion as landmark for the fine-tuning solely depends on the fact that it is clearly visible on US for the CIRS phantom we use. Despite its unlikeliness, in case no lesions at all can be detected on US images (one lesion is enough for this procedure), the fine-tuning process can be performed by tracking any other internal structure (like ducts or cysts). Likewise the calibration described above, optimal simulation parameters $\mathbf{p}^{*}$ breast are chosen as those minimizing the prediction error of our model (Equation 6), where in this case we only consider $n=1$ tracked landmark. Due to the fact that the calibration procedure on the ballistic gel phantom has given us a more precise idea of the range where optimal parameters lie, we perform the fine-tuning exploiting the direct search strategy implemented in MATLAB, which has proven convergence to local optimum and is more efficient than genetic algorithm, provided that it starts from a good initialization [27]. In particular, the starting parameter vector is initialized with optimal values obtained for the ballistic gel phantom $\left(\mathbf{p}^{*}{ }_{\text {gel }}\right)$ and the range for lower and upper bounds for each parameters is restricted to $40 \%$ of the initial range, centered in the starting point.

Once optimal parameters are found, the PBD model is updated and used to infer the displacement of each of the other 9 segmented lesions under four deformations, after the probe is moved such that the corresponding lesion can be seen on the US image.

\footnotetext{
1 https://gitlab.com/altairLab/breastsimulationpbd
} 


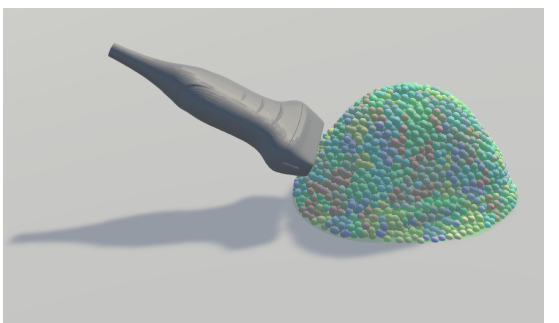

(a)

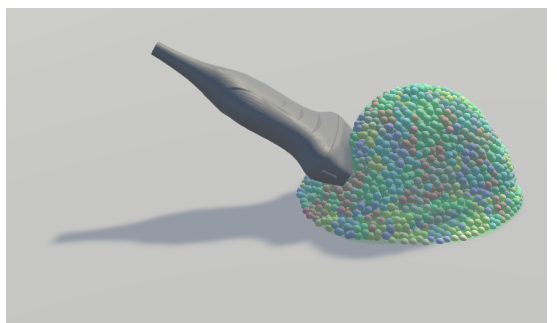

(b)

Fig. 4 PBD simulation of the CIRS phantom during the fine-tuning procedure: (a) before and (b) after probeinduced deformation. Different clusters are shown with different random colors

\section{Results}

The same convention is followed when reporting the results for both calibration and validation experiments. Lesion coordinates extracted on US images in the FUS coordinate system are considered as reference quantities. They are selected on US images as points belonging to bead/lesion contours. Localization errors are computed as Euclidean distance between these positions and the 3D coordinates of the PBD particle which, at rest, lies closer to the same location in the simulation scene. In particular, localization error at deformation $l$ relative to tumor $n$ is defined by:

$$
\varepsilon(l, n)=\left\|\mathbf{X}_{\mathbf{P B D}}(l, n)-\mathbf{X}_{\mathbf{U S}}(l, n)\right\|
$$

where ||.|| represents the Euclidean distance.

\subsection{Calibration}

This section reports the results relative to the calibration of simulation parameters on the ballistic gel phantom, and to their fine-tuning on the breast phantom. Table 2 reports optimal values for cluster spacing, radius and stiffness parameters estimated through the genetic algorithm strategy (for the calibration phantom) and the direct search method (for the breast phantom). The average error and standard deviation over all deformations, obtained when predicting the position of lesion 1 (the one selected as landmark for the fine-tuning process) when each set of parameters is used, is also tabulated. The fine-tuning process has allowed to achieve a reduction of $24 \%$ in the overall mean target error.

Table 2 Optimal values of cluster spacing, radius and stiffness parameters estimated with the proposed optimization strategies for the calibration and breast phantoms. Last columns report the mean error and standard deviation over all the deformations in $\mathrm{mm}$, when each set of parameters is used to predict the position of the landmark used for the fine-tuning process

\begin{tabular}{l|ccc|cc}
\hline & Cluster spacing & Cluster radius & Cluster stiffness & Mean Error & STD \\
\hline Calibration phantom & 9.6001 & 9.1674 & 0.452390 & 6.64 & 2.00 \\
\hline Breast phantom & 11.1626 & 8.5424 & 0.464890 & 5.07 & 1.62 \\
\hline
\end{tabular}




\subsection{Validation}

The PBD model initialized with the values estimated during the fine-tuning process is used to predict the displacement of all segmented inner lesions of the CIRS breast phantom, under four different input deformations. Model-predicted lesion positions can be projected onto US images in real-time, making it possible to track even those lesions which cannot be easily detected on US (Figure 5).

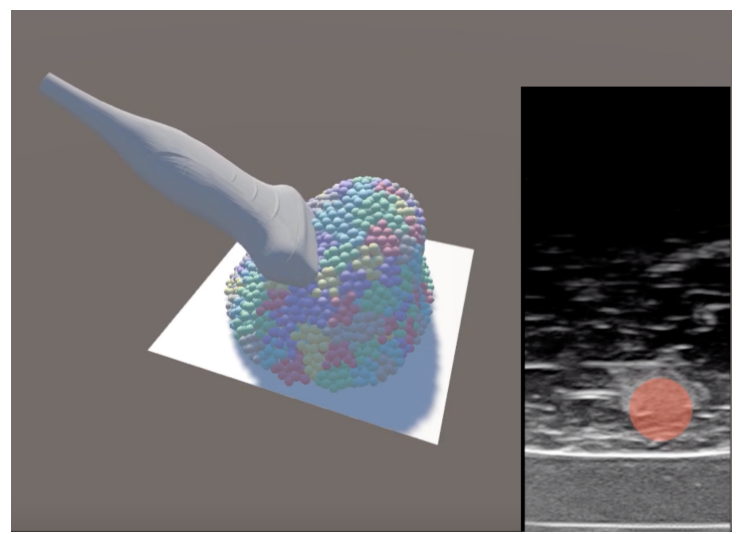

Fig. 5 The PBD model in the Unity scene (on the left) is used to predict lesion position due to US probe pressure. Updated lesion position is projected on the acquired US image in real-time as a red circular overlay. Circle dimension approximates the average lesion size

The performances of the proposed PBD approach are evaluated with respect to FE implementation of Neo-Hookean hyperelasticity provided by SOFA framework [28]. The choice of hyperelastic formulation is motivated by its popularity in breast biomechanical modelling and the fact that linear elasticity would not have been able to cope with the large input deformations applied. Young's modulus and Poisson's ratio are set in accordance with the values provided by Visentin et al., which are estimated on the same multimodal phantom we use in this work [29]. FE simulation is performed on a mesh of 26,220 linear tetrahedral elements, with the same boundary conditions used for the PBD model. The dynamic evolution of the system is obtained with an Euler implicit integration scheme, along with Pardiso solver to efficiently solve the large system of equations [30]. The only difference between the two scenarios follows from the way in which the input deformation is applied. Modelling probe-tissue interaction in a FE scenario as a contact problem introduces kinematic nonlinearities, which would make the nonlinear system of equilibrium equations even more demanding to solve. Therefore, instead of modeling the contacts explicitly, we prescribe the displacement of mesh nodes below the US probe to follow probe motion via penalty method. Coherently with the approach followed for the PBD model, localization errors are computed as Euclidean distance between reference landmarks extracted on US images and the closest node of the 3D FE mesh.

Table 3 shows the errors obtained for each phantom lesion as a function of the applied deformation, and the average error per tumor and per deformation. Input deformations are grouped in five ranges based on the displacements of US probe. In the Table, displacement ranges indicated with D15, D20 and D25 have fixed width of $5 \mathrm{~mm}$ and are centered in 15 , 
20 and $25 \mathrm{~mm}$ respectively. The two extreme ranges reported (D10 and D30) collect all the remaining values, i.e. D10 contains all the displacements $<12.5 \mathrm{~mm}$ while D30 contains all the values $>27.5 \mathrm{~mm}$. Data which are not acquired for some specific lesions are reported in Table 3 as missing values (-). The average time needed for the PBD model to predict anatomical deformations following the 4 input displacements is $6.99 \mathrm{~s}( \pm 0.36)$, which approximately corresponds to $1.75 \mathrm{~s}$ for simulating each input deformation. On the other hand, the FE model takes $16.37 \mathrm{~s}( \pm 0.73)$ on average, which corresponds to nearly $4.09 \mathrm{~s}$ for each input deformation. Real-time performances of the proposed method in predicting lesion position can be appreciated in the accompanying video (Online Resource 1).

Table 3 Mean localization error in $\mathrm{mm}$ for different tumors considering different deformations ranges in the breast phantom. The first table is for the proposed method, while the second table reports results obtained with the FE model

\begin{tabular}{c|ccccc|cc}
\multicolumn{8}{c}{ PBD method } \\
\hline TumorID & D10 & D15 & D20 & D25 & D30 & Mean & STD \\
\hline 1 & - & 3.259 & 3.724 & 5.307 & 6.214 & 4.626 & 1.190 \\
2 & 1.467 & 4.475 & 6.700 & - & 8.347 & 5.247 & 2.579 \\
3 & 3.689 & - & 5.590 & 7.831 & 11.930 & 7.260 & 3.069 \\
4 & 5.141 & 6.011 & 5.396 & 5.684 & - & 5.558 & 0.325 \\
5 & 2.190 & 2.018 & 4.501 & - & 6.694 & 3.851 & 1.912 \\
6 & 5.644 & 4.319 & 3.735 & - & 3.982 & 4.420 & 0.737 \\
7 & 2.810 & 3.961 & 6.374 & 10.636 & - & 5.945 & 2.998 \\
8 & 5.581 & 5.659 & 6.120 & 6.683 & - & 6.011 & 0.440 \\
9 & - & 4.506 & 3.833 & 4.007 & 4.511 & 4.214 & 0.301 \\
10 & 4.606 & 2.990 & 3.193 & 3.774 & - & 3.641 & 0.627 \\
\hline Mean & 3.891 & 4.133 & 4.917 & 6.274 & 6.946 & & \\
STD & 1.499 & 1.189 & 1.207 & 2.213 & 2.649 & & \\
\hline
\end{tabular}

\begin{tabular}{c|ccccc|cc}
\multicolumn{8}{c}{ FE method } \\
\hline TumorID & D10 & D15 & D20 & D25 & D30 & Mean & STD \\
\hline 1 & - & 2.686 & 2.495 & 3.626 & 5.175 & 3.495 & 1.060 \\
2 & 3.621 & 5.964 & 5.804 & - & 7.087 & 5.619 & 1.255 \\
3 & 3.793 & - & 6.952 & 8.136 & 9.646 & 7.132 & 2.151 \\
4 & 4.142 & 3.886 & 4.954 & 4.822 & - & 4.451 & 0.449 \\
5 & 1.255 & 1.275 & 1.590 & - & 3.758 & 1.970 & 1.041 \\
6 & 6.581 & 5.871 & 6.410 & - & 6.671 & 6.383 & 0.310 \\
7 & 5.462 & 5.614 & 6.414 & 10.768 & - & 7.065 & 2.169 \\
8 & 6.983 & 4.835 & 4.874 & 5.418 & - & 5.527 & 0.871 \\
9 & - & 5.032 & 5.277 & 5.047 & 6.503 & 5.465 & 0.607 \\
10 & 2.682 & 2.831 & 3.341 & 2.966 & - & 2.955 & 0.245 \\
\hline Mean & 4.315 & 4.222 & 4.811 & 5.826 & 6.473 & & \\
STD & 1.816 & 1.557 & 1.698 & 2.523 & 1.805 & & \\
\hline
\end{tabular}

Figures 6 and 7 compare the performances of the proposed deformation model with the FE model and a rigid one. Since deformations are not accounted for in the rigid scenario, errors relative to the rigid case are computed as difference between the lesion position at rest (which always corresponds to the predicted position) and the real lesion position, both identified on US images. 


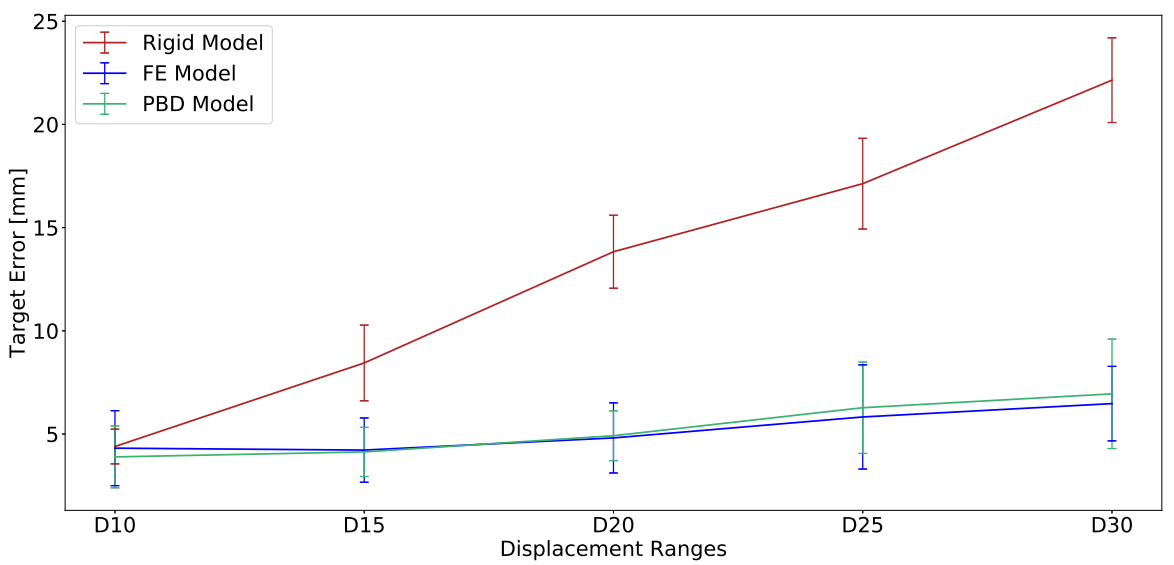

Fig. 6 Average lesions localization errors in $\mathrm{mm}$ at different levels of applied deformations (in $\mathrm{mm}$ ), for rigid (red), PBD (green) and FE (blue) models.

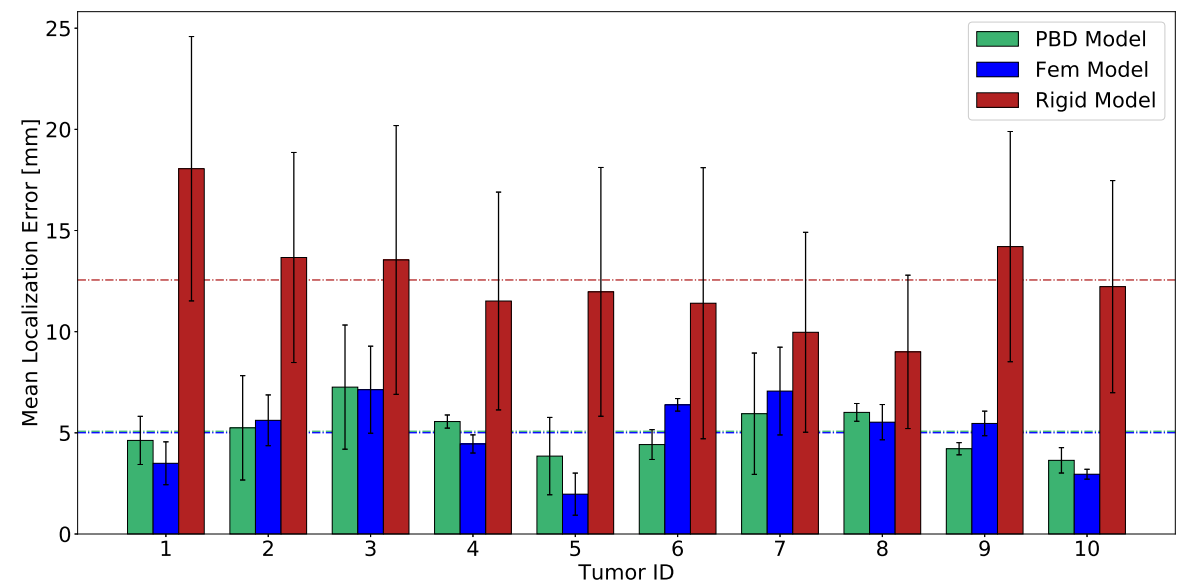

Fig. 7 Mean localization error in mm obtained for each tumor for rigid (red), PBD (green) and FE (blue) models. Horizontal dashed lines represent the corresponding average error

\section{Discussion}

This paper presents a biomechanical model able to account for the dynamic behavior of the breast during US scanning. The preliminary calibration of the main deformation parameters on a distinct geometry serves to find reasonable initialization values and can be performed offline once for all. Before applying the deformation model, simulation parameters are refined with a fine-tuning procedure on the final structure of interest, in order to improve parameter values to describe patient-specific features. Even though the fine-tuning process is able to further optimize the parameters, Table 2 shows that errors obtained with the ini- 
tial set can be considered already acceptable. We expect that once the model is applied to the true clinical context, where the high inter-patient variability in geometries and boundary conditions is unlikely to be described by a single phantom, the role of the fine-tuning will emerge more clearly.

The performance of the PBD model in the prediction of the displacement of internal lesions is also tested. From Table 3 it is possible to evince that for all the inner masses, the average prediction error over all the deformations (second to last column in the Table 3 ) remains below $7.26 \mathrm{~mm}$, which is comparable to the maximum average error made by the FE model $(7.13 \mathrm{~mm})$. If we consider the average error per deformation level, it emerges that while PBD model performs better for smaller input displacements (D10 and D15), FEM has improved performances with larger displacements (D20 to D30). It is well-known that accuracy of FE results would benefit from using a higher mesh resolution, but this would come at the expense of a degradation in computation time [7]. Due to the fact that the main aim of this work is to update lesion position on ultrasounds in real-time for tracking purposes, comparison with a highly refined FE mesh has not been considered in this work. In general, Table 3 clearly shows that there are no significant differences in the errors made by the two models, thus suggesting that the prediction accuracy obtained with PBD model is comparable to that achieved with classical FEM, which is typically used to simulate soft tissue biomechanics. If we consider the trend of the error at different deformation levels (Figure 6), it is possible to immediately notice that performances of the PBD and FE models are highly comparable. In particular, they are both able to keep the prediction error limited even at larger deformations, where the rigid case considerably fails. Instead, for small deformations, all the three models perform in a similar way. Figure 7 allows to analyze the prediction performance of the deformation model over all the tumors. It is immediate to notice that both PBD and FE models outperform the rigid case by at least halvening the prediction error on all the tumors. No remarkable differences between results obtained for the two deformable models can be noticed, with the PBD model performing better than FEM on 6 out of 10 lesions, on average. The biggest average error is obtained for both models for tumor 3 , the closest to the phantom base, possibly due to an inaccuracy of the models in tackling lesions in that position (i.e. very close to the boundaries).

Analyzing the computational performances, FE model takes more than twice the time needed by the proposed PBD model to perform the simulation, and even without any significant improvement in prediction accuracy. Although a wide variety of techniques have been proposed to simplify the computational complexity of FEM to meet real-time performances [7], we compare our method with the implementation provided by the SOFA framework, which is the state-of-the-art physics engine for interactive medical applications and it is freely available. In addition, SOFA has been already employed to model probeinduced deformation of soft tissues [31]. An extensive comparison with more advanced FE implementations will be considered in future work. However, apart from the computational performace, the proposed PBD model has several advantages over FEM. First of all, the mesh-free PBD approach allows to avoid the time-consuming generation of high quality mesh, which represents the major bottleneck in FE simulations (especially in those inolving large deformations) [7]. Since we are targeting a patient-specific context, this represents an enormous advantage because the mesh would have to be constructed everytime, for each patient. Furthermore, thanks to its direct manipulation of positions, the PBD approach can easily handle collisions constraints. Probe-tissue interaction can thus be effectively treated as a collision problem, thus allowing to deal with any input probe position without requiring the explicit definition of the contacting surface. The same does not apply to FE simulations, 
where the enforcement of contact constraints would introduce a degradation of the performances and stability issues.

The proposed approach relies on the region-based shape matching constraint to model large deformations of soft tissues. The main drawbacks of this implementation are the dependence of the deformable behavior on time step size and iteration count, and the fact that PBD parameters do not have a direct physical meaning. Some advanced approaches that overcome these limitations have been proposed but they are not yet incorporated in Nvidia FleX framework and not even publicly available. In addition, they have never demonstrated to be significantly faster than the shape matching method even in its CPU version, so we can reasonably assume that the optimized GPU-based implementation of Nvidia FleX outperforms both approaches from a computational time point of view [32, 9]. In future works, we plan to compare the performances of the proposed method with these extensions and also with other approaches such as mass-spring models, ChainMail and smooth particle hydrodynamics (SPH).

The most innovative aspect of the proposed model relies in its ability to compensate in real-time for the large deformations the breast is subject to due to probe pressure during freehand US acquisitions, thus enabling a precise tracking of biopsy targets. However, this method still has some margin of improvement. First of all, a more controllable and repeatable strategy for the application of deformations should be envisioned, for example by using a probe holder or by performing robotic-assisted acquisition. To further reduce inaccuracies, a more precise selection of corresponding fiducials in real and simulated environments has to be made. For instance, an automatic routine for fiducials identification on US images would allow to avoid human errors involved in the landmarks placement. Regarding the simulated environment, it would be optimal either to increment the total number of particles (thus increasing the degrees of freedom) or to force a particle to lie at the same exact location of the real fiducial. However, both particle amount and their placement in space are handled by the current Unity implementation (optimized for gaming applications) and cannot be controlled by the user. As a further extension, we will provide a complete tool for guiding biopsies by including needle insertion simulation. Despite the advantages provided by a meshless approach in handling topology changes, we expect some major challenges in the modelling of needle-tissue interaction. Afterwards, we envision to apply the same method to improve the effectiveness of US-guidance in other percutaneous procedures, such as prostate biopsy.

\section{Conclusion}

Exploiting position-based dynamics formulation for modelling breast deformations has proved successful to online predict probe-induced displacement of internal lesions during ultrasound scanning. By accounting for the deformable nature of the anatomy, the proposed approach achieves accuracy which is comparable with FE models, but with faster computational performance and without even requiring 3D mesh generation. Furthermore, it outperforms rigid models usually employed for lesion tracking in biopsy procedures, and paves the way to a wider range of applications, such as planning of optimal transducer trajectories in robotic-assisted US scanning and realistic ultrasound simulation for training purposes.

Acknowledgements This project has received funding from the European Research Council (ERC) under the European Union's Horizon 2020 research and innovation programme (grant agreement No 742671 "ARS" and No 688188 "MURAB"). 
Compliance with ethical standards

Conflict of Interest The authors declare that they have no conflict of interest.

Ethical approval This article does not contain any studies with human participants or animals performed by any of the authors.

\section{References}

1. O’Flynn E, Wilson A, Michell M (2010) Image-guided breast biopsy: state-of-the-art. Clinical radiology 65(4):259-270

2. Apesteguía L, Pina LJ (2011) Ultrasound-guided core-needle biopsy of breast lesions. Insights into imaging 2(4):493-500

3. Guo R, Lu G, Qin B, Fei B (2017) Ultrasound imaging technologies for breast cancer detection and management: A review. Ultrasound in medicine \& biology

4. Kucukkaya F, Aribal E, Tureli D, Altas H, Kaya H (2016) Use of a volume navigation technique for combining real-time ultrasound and contrast-enhanced mri: accuracy and feasibility of a novel technique for locating breast lesions. American Journal of Roentgenology 206(1):217-225

5. Aribal E, Tureli D, Kucukkaya F, Kaya H (2017) Volume navigation technique for ultrasound-guided biopsy of breast lesions detected only at mri. American Journal of Roentgenology 208(6):1400-1409

6. Hipwell JH, Vavourakis V, Han L, Mertzanidou T, Eiben B, Hawkes DJ (2016) A review of biomechanically informed breast image registration. Physics in Medicine \& Biology 61(2):R1, URL http://stacks . iop.org/0031-9155/61/i=2/a=R1

7. Zhang J, Zhong Y, Gu C (2018) Deformable models for surgical simulation: A survey. IEEE reviews in biomedical engineering 11:143-164

8. Bender J, Müller M, Macklin M (2017) A survey on position based dynamics, 2017. EUROGRAPHICS 2017 Tutorials

9. Bender J, Koschier D, Charrier P, Weber D (2014) Position-based simulation of continuous materials. Computers \& Graphics 44:1-10

10. Rodero C, Real P, Zuñeda P, Monteagudo C, Lozano M, García-Fernández I (2016) Characterisation of position based dynamics for elastic materials. In: Proceedings of the XXVI Spanish Computer Graphics Conference, Eurographics Association, pp 4957

11. Berndt I, Torchelsen R, Maciel A (2017) Efficient surgical cutting with position-based dynamics. IEEE computer graphics and applications 38(3):24-31

12. Pan J, Bai J, Zhao X, Hao A, Qin H (2015) Real-time haptic manipulation and cutting of hybrid soft tissue models by extended position-based dynamics. Computer Animation and Virtual Worlds 26(3-4):321-335

13. Wang Y, Xiong Y, Xu K, Tan K, Guo G (2006) A mass-spring model for surface mesh deformation based on shape matching. In: GRAPHITE, vol 6, pp 375-380

14. Xu L, Lu Y, Liu Q (2018) Integrating viscoelastic mass spring dampers into positionbased dynamics to simulate soft tissue deformation in real time. Royal Society open science 5(2): 171,587

15. Kubiak B, Pietroni N, Ganovelli F, Fratarcangeli M (2007) A robust method for realtime thread simulation. In: Proceedings of the 2007 ACM symposium on Virtual reality software and technology, ACM, pp 85-88 
16. Camara M, Mayer E, Darzi A, Pratt P (2017) Simulation of patient-specific deformable ultrasound imaging in real time. In: Imaging for Patient-Customized Simulations and Systems for Point-of-Care Ultrasound, Springer, pp 11-18

17. Camara M, Mayer E, Darzi A, Pratt P (2016) Soft tissue deformation for surgical simulation: a position-based dynamics approach. International journal of computer assisted radiology and surgery 11(6):919-928

18. Müller M, Heidelberger B, Teschner M, Gross M (2005) Meshless deformations based on shape matching. In: ACM transactions on graphics (TOG), ACM, vol 24, pp 471-478

19. (2018) NVIDIA gameworks. Nvidia FleX. https ://developer.nvidia.com/flex, [Accessed: 2018-12-20]

20. Lasso A, Heffter T, Rankin A, Pinter C, Ungi T, Fichtinger G (2014) Plus: open-source toolkit for ultrasound-guided intervention systems. IEEE Transactions on Biomedical Engineering 61(10):2527-2537

21. Fedorov A, Beichel R, Kalpathy-Cramer J, Finet J, Fillion-Robin JC, Pujol S, Bauer C, Jennings D, Fennessy F, Sonka M, Buatti J, Aylward S, Miller J, Pieper S, Kikinis R (2012) 3d slicer as an image computing platform for the quantitative imaging network. Magnetic resonance imaging 30(9):1323-1341

22. Tokuda J, Fischer GS, Papademetris X, Yaniv Z, Ibanez L, Cheng P, Liu H, Blevins J, Arata J, Golby AJ, Kapur T, Pieper S, Burdette E, Fichtinger G, Tempany C, Hata N (2009) Openigtlink: an open network protocol for image-guided therapy environment. The International Journal of Medical Robotics and Computer Assisted Surgery 5(4):423-434

23. Amini R, Kartchner JZ, Stolz LA, Biffar D, Hamilton AJ, Adhikari S (2015) A novel and inexpensive ballistic gel phantom for ultrasound training. World journal of emergency medicine 6(3):225

24. Mitchell M (1998) An introduction to genetic algorithms. MIT press

25. Yushkevich PA, Piven J, Cody Hazlett H, Gimpel Smith R, Ho S, Gee JC, Gerig G (2006) User-guided 3D active contour segmentation of anatomical structures: Significantly improved efficiency and reliability. Neuroimage 31(3):1116-1128

26. Cignoni P, Callieri M, Corsini M, Dellepiane M, Ganovelli F, Ranzuglia G (2008) MeshLab: an Open-Source Mesh Processing Tool. In: Scarano V, Chiara RD, Erra U (eds) Eurographics Italian Chapter Conference, The Eurographics Association, DOI 10.2312/LocalChapterEvents/ItalChap/ItalianChapConf2008/129-136

27. Audet C, Dennis Jr JE (2002) Analysis of generalized pattern searches. SIAM Journal on optimization 13(3):889-903

28. Faure F, Duriez C, Delingette H, Allard J, Gilles B, Marchesseau S, Talbot H, Courtecuisse H, Bousquet G, Peterlik I, et al (2012) Sofa: A multi-model framework for interactive physical simulation. In: Soft tissue biomechanical modeling for computer assisted surgery, Springer, pp 283-321

29. Visentin F, Groenhuis V, Maris B, DallAlba D, Siepel F, Stramigioli S, Fiorini P (2018) Iterative simulations to estimate the elastic properties from a series of mri images followed by mri-us validation. Medical \& biological engineering \& computing pp 1-12

30. Schenk O, Gärtner K (2004) Solving unsymmetric sparse systems of linear equations with pardiso. Future Generation Computer Systems 20(3):475-487

31. Moreira P, Peterlik I, Herink M, Duriez C, Cotin S, Misra S (2013) Modelling prostate deformation: Sofa versus experiments. Prostate 17(83.0):1-0

32. Macklin M, Müller M, Chentanez N (2016) Xpbd: position-based simulation of compliant constrained dynamics. In: Proceedings of the 9th International Conference on Motion in Games, ACM, pp 49-54 